\title{
CROSS-RATIO DISTORTION AND DOUADY-EARLE EXTENSION: III. HOW TO CONTROL THE DILATATION NEAR THE ORIGIN
}

\author{
Jun $\mathrm{Hu}^{*}$ and Oleg Muzician \\ Brooklyn College of CUNY, Department of Mathematics \\ Brooklyn, NY 11210, U.S.A.; junhu@brooklyn.cuny.edu \\ and Graduate Center of CUNY, Ph.D. Program in Mathematics \\ 365 Fifth Avenue, New York, NY 10016; JHu1@gc.cuny.edu \\ CUNY BMCC, Department of Mathematics \\ 199 Chambers Street, New York, NY 10007, U.S.A; OMuzician@bmcc.cuny.edu
}

\begin{abstract}
In this paper, we study how the maximal dilatation of the Douady-Earle extension near the origin is controlled by the distortion of the boundary map on finitely many points. Consider the case of points evenly spread on the circle. We show that the maximal dilatation of the extension in a neighborhood of the origin has an upper bound only depending on the cross-ratio distortion of the boundary map on these points if and only if the number $n$ of the points is more than 4 . Furthermore, we show that the size of the neighborhood is universal for each $n \geq 5$ in the sense that its size only depends on the distortion.
\end{abstract}

\section{Introduction}

Let $\mathbf{S}^{1}$ be the unit circle and $n$ a positive integer. We define $\mathcal{F}_{n}$ to be the collection of the homeomorphisms of $\mathbf{S}^{1}$ fixing $n$ points evenly spread on $\mathbf{S}^{1}$. Let $\Phi(f)$ be the Douady-Earle extension of $f$ to the unit disk $\mathbf{D}$ and $K(\Phi(f))(0)$ be the maximal dilatation of $\Phi(f)$ at the origin 0 of $\mathbf{D}$. Consider

$$
\sup _{f \in \mathcal{F}_{n}} K(\Phi(f))(0) \text {. }
$$

One may easily observe the following. On one hand, the supremum is infinite if $n=1,2$ or 3 . On the other hand, if $n$ is very large, then each element $f$ of $\mathcal{F}_{n}$ is quite close to the identity map in the $C^{0}$ topology. It follows that the DouadyEarle extension $\Phi(f)$ is close to the identity in the $C^{\infty}$ topology on $\mathbf{D}$ and hence the maximal dilatation of $\Phi(f)$ at 0 is close to the one of the identity map. This means the supremum is close to 1 when $n$ is large enough. An interesting question arise: what is the minimum value of $n$ such that the supremum is finite? In this paper, we first show that this supremum is finite if and only if $n \geq 5$. Furthermore, we prove that there exists a neighborhood $U$ of the origin in $\mathbf{D}$ such that for each $n \geq 5$,

$$
\sup _{f \in \mathcal{F}_{n}} K\left(\left.\Phi(f)\right|_{U}\right)<\infty .
$$

This property implies that for each $n \geq 5,\left\{\left.\Phi(f)\right|_{U}\right\}_{f \in \mathcal{F}_{n}}$ is a normal family under certain normalization.

To prove these two results, it suffices to show the following theorem.

https://doi.org/10.5186/aasfm.2019.4432

2010 Mathematics Subject Classification: Primary 30C62, 30F60.

Key words: Douady-Earle extension, quasisymmetric homeomorphism, cross-ratio distortion, extremal quasiconformal extension, simple earthquake map.

*The research is partially supported by PSC-CUNY research awards. 
Theorem 1. (i) There exists a (universal) neighborhood $U$ of the origin in D such that

$$
\sup _{n \geq 5} \sup _{f \in \mathcal{F}_{n}} K\left(\left.\Phi(f)\right|_{U}\right)<\infty,
$$

where $K\left(\left.\Phi(f)\right|_{U}\right)$ denotes the maximal dilatation of $\Phi(f)$ on $U$.

(ii) For each $n<5$,

$$
\sup _{f \in \mathcal{F}_{n}} K(\Phi(f))(0)=\infty
$$

When studying regularities of Douady-Earle extensions, one often normalizes them to fix the origin; that is, $\Phi(f)(0)=0$. This motivates us to consider a subset of $\mathcal{F}_{n}$. We define $\mathcal{F}_{n}^{0}$ to be the subset of $\mathcal{F}_{n}$ such that the Douady-Earle extensions of the maps in $\mathcal{F}_{n}^{0}$ fix the origin. Now we consider the same supremum problem for $\mathcal{F}_{n}^{0}$. Clearly, the first part of Theorem 1 holds for this subset, but the conclusion of the second part changes when $n=3$. We obtain the following theorem.

Theorem 2. (i) There exists a universal neighborhood $U$ of the origin in $\mathbf{D}$ such that

$$
\sup _{f \in \mathcal{F}_{3}^{0}} K\left(\left.\Phi(f)\right|_{U}\right)<\infty
$$

(ii) For each $n=1,2,4$,

$$
\sup _{f \in \mathcal{F}_{n}^{0}} K(\Phi(f))(0)=\infty .
$$

In the second half of the paper, we use cross-ratio distortion to relax the condition(s) on the homeomorphisms $f$ in $\mathcal{F}_{n}$ with $n \geq 5$ and obtain similar upper bound properties for collections of circle homeomorphisms more general than the ones in $\mathcal{F}_{n}$. We first introduce some concepts and notation.

Given a quadruple $Q=\{a, b, c, d\}$ consisting of four points $a, b, c, d$ on the unit circle $\mathbf{S}^{1}$ arranged in counterclockwise order, we define a cross ratio of $Q$ by

$$
\operatorname{cr}(Q)=\frac{(b-a)(d-c)}{(c-b)(d-a)} .
$$

It is easy to check that a quadruple $Q$ has $\operatorname{cr}(Q)=1$ if and only if the geodesic $\overline{a c}$ from $a$ to $c$ is perpendicular to the geodesic $\overline{b d}$ from $b$ to $d$.

Given an orientation-preserving homeomorphism $f$ of $\mathbf{S}^{1}$, a cross-ratio distortion norm of $f$ is defined as

$$
\|f\|_{c r}=\sup _{\operatorname{cr}(Q)=1}|\ln c r(f(Q))|
$$

where

$$
c r(f(Q))=\frac{(f(b)-f(a))(f(d)-f(c))}{(f(c)-f(b))(f(d)-f(a))} .
$$

In this paper, we need to consider quadruples $Q$ with $\operatorname{cr}(Q) \neq 1$. For each constant $L \geq 1$, we define a cross-ratio distortion norm of $f$ to be

$$
\|f\|_{c r}^{L}=\sup _{\frac{1}{L} \leq c r(Q) \leq L}\left|\ln \frac{c r(f(Q))}{c r(Q)}\right| .
$$

Clearly, $\|f\|_{c r}^{L}$ is invariant under pre or post composition by any Möbius transformation preserving the unit disk. 
One often defines $f$ to be quasisymmetric if there exists a positive constant $M$ such that for any $0 \leq t<2 \pi$ and $0<s<\pi$,

$$
\frac{1}{M} \leq \frac{\left|f\left(e^{i(t+s)}\right)-f\left(e^{i t}\right)\right|}{\left|f\left(e^{i t}\right)-f\left(e^{i(t-s)}\right)\right|} \leq M .
$$

It is known that $f$ is quasisymmetric if and only if $\|f\|_{c r}^{L}$ is finite for some $L \geq 1$. On the other hand, if one uses the following supremum

$$
\|f\|_{c r}^{\infty}=\sup _{Q}\left|\ln \frac{c r(f(Q))}{c r(Q)}\right|
$$

then the quasisymmetry of $f$ is not equivalent to $\|f\|_{c r}^{\infty}<\infty$. Although $\|f\|_{c r}^{\infty}<\infty$ implies $f$ to be quasisymmetric, the converse is not true. This means there exist quasisymmetric homeomorphisms $f$ of $\mathbf{S}^{1}$ with $\|f\|_{c r}^{\infty}=\infty$. For example, consider conjugations of homeomorphisms $g(x)=\operatorname{sign}(x)|x|^{\alpha}$ of the real line to $\mathbf{S}^{1}$, where $\alpha>0$ but $\neq 1$. In this paper, we only use this notation for $f$ when restricted on a finite set of points on $\mathbf{S}^{1}$.

For each positive constant $M \geq 0$ and each positive integer $n \geq 4$, a collection $\mathcal{B}_{n}^{M}$ of homeomorphisms $f$ of $\mathbf{S}^{1}$ is defined as follows: $f \in \mathcal{B}_{n}^{M}$ if there exists a set $S_{n}$ of $n$ points evenly distributed on $\mathbf{S}^{1}$ such that $\left\|\left.f\right|_{S_{n}}\right\|_{c r}^{\infty} \leq M$, where

$$
\left\|\left.f\right|_{S_{n}}\right\|_{c r}^{\infty}=\sup _{Q \subset S_{n}}\left|\ln \frac{c r(f(Q))}{c r(Q)}\right| \text {. }
$$

Clearly,

$$
\mathcal{F}_{n} \subset \mathcal{B}_{n}^{0} \subset \mathcal{B}_{n}^{M}
$$

for each $n \geq 4$ and any $M \geq 0$. An immediate corollary of Part (ii) of Theorem 1 follows.

\section{Corollary 1.}

$$
\sup _{f \in \mathcal{B}_{4}^{M}} K(\Phi(f))(0)=\infty .
$$

We also obtain the following result.

Theorem 3. For each integer $n \geq 5$ and any constant $M \geq 0$, there exists a neighborhood $U$ of the origin such that

$$
\sup _{f \in \mathcal{B}_{n}^{M}} K\left(\left.\Phi(f)\right|_{U}\right)<\infty
$$

where the supremum and the size of $U$ depend on $n$ and $M$.

In fact, a more general and even better result of Theorem 3 can be developed. Given $L \geq 1, M \geq 0$ and a positive integer $n$, a collection $\mathcal{A}_{n}^{L, M}$ of circle homeomorphisms $f$ is defined as follows: $f \in \mathcal{A}_{n}^{L, M}$ if there exists a set $S_{n}$ of $n$ points evenly distributed on $\mathbf{S}^{1}$ such that $\left\|\left.f\right|_{S_{n}}\right\|_{c r}^{L} \leq M$, where

$$
\left\|\left.f\right|_{S_{n}}\right\|_{c r}^{L}=\sup _{Q \subset S_{n}, \frac{1}{L} \leq c r(Q) \leq L}\left|\ln \frac{\operatorname{cr}(f(Q))}{c r(Q)}\right| .
$$

We prove the following theorem.

Theorem 4. There exists a constant $L>1$ such that for any $M \geq 0$, there exists a neighborhood $U$ of the origin such that

$$
\sup _{n \geq 5} \sup _{f \in \mathcal{A}_{n}^{L, M}} K\left(\left.\Phi(f)\right|_{U}\right)<\infty
$$


where the neighborhood $U$ and the supremum only depend on $M$.

Since $\mathcal{B}_{n}^{M} \subset \mathcal{A}_{n}^{L, M}$ for any constant $L \geq 1$, Theorem 4 implies Theorem 3 . We also say that Theorem 4 is stronger than Theorem 3 in the sense that the size of $U$ in Theorem 4 is independent of $n$.

The paper is arranged as follows. In Section 2, we give a short overview of known results on Douady-Earle extensions of circle homeomorphisms. Then we prove Theorems 1 and 2 in Section 3. Finally, we prove Theorem 4 in Section 4.

\section{Some background on the Douady-Earle extension}

Let $f$ be an orientation-preserving homeomorphism on the unit circle $\mathbf{S}^{1}$. The Douady-Earle extension $\Phi(f)$ of $f$ is defined as follows. Let $\mathbf{D}$ be the open unit disk centered at the origin. Given a point $z \in \mathbf{D}$, let $\eta_{z}$ be the harmonic measure on $\mathbf{S}^{1}$ viewed from $z$; that is, for any Borel set $A \subset \mathbf{S}^{1}$,

$$
\eta_{z}(A)=\frac{1}{2 \pi} \int_{A} \frac{1-|z|^{2}}{|z-\xi|^{2}}|d \xi| .
$$

Now let $f_{*}\left(\eta_{z}\right)$ be the push-forward of the measure $\eta_{z}$ by $f$; that is, $f_{*}\left(\eta_{z}\right)(A)=$ $\eta_{z}\left(f^{-1}(A)\right)$ for any Borel set $A \subset \mathbf{S}^{1}$. It is shown in [3] that there exists a unique $w \in \mathbf{D}$ such that

$$
\frac{1}{2 \pi} \int \frac{\zeta-w}{1-\bar{w} \zeta} d f_{*}\left(\eta_{z}\right)(\zeta)=0
$$

which is called the conformal barycenter for the measure $f_{*}\left(\eta_{z}\right)$ and denoted by $w=B\left(f_{*}\left(\eta_{z}\right)\right.$ ) (it can be approximated through an iterated algorithm developed in [1]). Finally the Douady-Earle extension $\Phi(f)$ of $f$ is defined as: $\Phi(f)(z)=B\left(f_{*}\left(\eta_{z}\right)\right)$ for each $z \in \mathbf{D}$ and $\Phi(f)(z)=f(z)$ for each $z \in \mathbf{S}^{1}$.

In summary, given any point $z \in \mathbf{D}, \Phi(f)(z)$ is defined to be the unique point $w \in \mathbf{D}$ such that

$$
F(z, w)=0
$$

where

$$
F(z, w)=\frac{1}{2 \pi} \int_{\mathbf{S}^{1}} \frac{f(\xi)-w}{1-\bar{w} f(\xi)} \cdot \frac{1-|z|^{2}}{|z-\xi|^{2}}|d \xi| .
$$

The extension $\Phi(f)$ has the following two important features: (1) $\Phi(f)$ is an orientation-preserving homeomorphism on the closed disk $\overline{\mathbf{D}}$ and a real analytic diffeomorphism on $\mathbf{D} ;(2) \Phi(f)$ is conformally natural in the sense that for any two conformal homeomorphisms $A$ and $B$ of $\mathbf{D}, \Phi(A \circ f \circ B)=A \circ \Phi(f) \circ B$. For proofs for these statements, we refer to [3]. Conformally natural extensions were developed in [2] for monotone maps of the circle, in [7] for circle endomorphisms, and in [8] for general continuous circle maps.

By using the conformal naturality of $\Phi(f)$, the work of estimating the complex dilatation of $\Phi(f)$ is reduced to estimating the complex dilatation at the origin for a normalized map $\Phi(f)$; that is, $\Phi(f)(0)=0$. Let $\Phi(f)$ be an extension with such a normalization. By the differentiability of $\Phi(f)$, the Beltrami coefficient of $\Phi(f)$ can be explicitly expressed in terms of the partial derivatives of $F$ at the origin $(0,0)$. If we denote by

$$
c_{1}=\frac{\partial F}{\partial z}(0,0)=\frac{1}{2 \pi} \int_{\mathbf{S}^{1}} \bar{\xi} f(\xi)|d \xi|, \quad c_{-1}=\frac{\partial F}{\partial \bar{z}}(0,0)=\frac{1}{2 \pi} \int_{\mathbf{S}^{1}} \xi f(\xi)|d \xi|
$$


and

$$
d_{1}=\frac{\partial F}{\partial w}(0,0)=-1, \quad d_{-1}=\frac{\partial F}{\partial \bar{w}}(0,0)=\frac{1}{2 \pi} \int_{\mathbf{S}^{1}} f(\xi)^{2}|d \xi|
$$

then

$$
\frac{\partial w}{\partial \bar{z}}(0)=-\frac{\overline{\frac{\partial F}{\partial w}(0,0)} \frac{\partial F}{\partial \bar{z}}(0,0)-\frac{\partial F}{\partial \bar{w}}(0,0) \frac{\overline{\partial F}(0,0)}{\partial z}}{\left|\frac{\partial F}{\partial w}(0,0)\right|^{2}-\left|\frac{\partial F}{\partial \bar{w}}(0,0)\right|^{2}}
$$

and

$$
\frac{\partial w}{\partial z}(0)=-\frac{\overline{\frac{\partial F}{\partial w}(0,0)} \frac{\partial F}{\partial z}(0,0)-\frac{\partial F}{\partial \bar{w}}(0,0) \overline{\frac{\partial F}{\partial \bar{z}}(0,0)}}{\left|\frac{\partial F}{\partial w}(0,0)\right|^{2}-\left|\frac{\partial F}{\partial \bar{w}}(0,0)\right|^{2}} .
$$

Furthermore, the Jacobian of $\Phi(f)$ at 0 is equal to

$$
\left|\frac{\partial w}{\partial z}(0)\right|^{2}-\left|\frac{\partial w}{\partial \bar{z}}(0)\right|^{2}=\frac{\left|\frac{\partial F}{\partial z}(0,0)\right|^{2}-\left|\frac{\partial F}{\partial \bar{z}}(0,0)\right|^{2}}{\left|\frac{\partial F}{\partial w}(0,0)\right|^{2}-\left|\frac{\partial F}{\partial \bar{w}}(0,0)\right|^{2}}=\frac{\left|c_{1}\right|^{2}-\left|c_{-1}\right|^{2}}{\left|d_{1}\right|^{2}-\left|d_{-1}\right|^{2}} .
$$

Now let $h: \mathbf{R} \rightarrow \mathbf{R}$ be a lifting of $f$ to the real line $\mathbf{R}$; that is, $f\left(e^{i u}\right)=e^{i h(u)}$ for any $u \in \mathbf{R}$, where $h(u+2 \pi)=h(u)+2 \pi$. Then one can rewrite

$$
\left|d_{1}\right|^{2}-\left|d_{-1}\right|^{2}=2\left(\frac{1}{2 \pi}\right)^{2} \int_{0}^{2 \pi} \int_{0}^{2 \pi} \sin ^{2}(h(s)-h(t)) d s d t .
$$

It follows that $\left|d_{1}\right|^{2}-\left|d_{-1}\right|^{2}>0$.

In [3], $\left|c_{1}\right|^{2}-\left|c_{-1}\right|^{2}$ is expressed as

$$
\left|c_{1}\right|^{2}-\left|c_{-1}\right|^{2}=\left(\frac{1}{2 \pi}\right)^{2} \int_{u=0}^{\pi} \sin u \int_{t=0}^{2 \pi} H(t, u) d t d u
$$

with

$$
\begin{aligned}
H(t, u)= & \sin (h(t+u)-h(t))+\sin (h(t+2 \pi)-h(t+u+\pi)) \\
& +\sin (h(t+\pi+u)-h(t+\pi))+\sin (h(t+\pi)-h(t+u)) .
\end{aligned}
$$

Let $\alpha_{1}=h(t+u)-h(t), \alpha_{2}=h(t+2 \pi)-h(t+u+\pi), \alpha_{3}=h(t+\pi+u)-h(t+\pi)$ and $\alpha_{4}=h(t+\pi)-h(t+u)$. Clearly, all $\alpha_{j}$ 's are nonnegative and their sum $\sum \alpha_{j}=2 \pi$. By applying the summation formula from trigonometry, it is obtained in [2] that

$$
\sum_{j=1}^{4} \sin \alpha_{j}=4 \sin \frac{\alpha_{1}+\alpha_{2}}{2} \sin \frac{\alpha_{1}+\alpha_{3}}{2} \sin \frac{\alpha_{2}+\alpha_{3}}{2} .
$$

Now we can easily see $\left|c_{1}\right|^{2}-\left|c_{-1}\right|^{2}>0$ since $H(t, u) \geq 0$ for all $t$ and $u$ and is not identically 0 . It follows that the Jacobian of $\Phi(f)$ at the origin is positive. By the conformal naturality, the Jacobian of $\Phi(f)$ is positive at every point $z \in \mathbf{D}$, which implies that $\Phi(f)$ is an orientation-preserving homeomorphism on $\mathbf{D}([3])$. Furthermore, $\Phi(f)$ is quasiconformal if $f$ admits a quasiconformal extension ([3]). It is proved in [5] that the maximal dilatation $K(\Phi(f))$ on the unit disk $\mathbf{D}$ depends on $\|f\|_{c r}$ in a linear fashion.

Using the expressions (9)-(16), Markovic developed a criterion (Lemma 3.6, [9]) for a family of circle homeomorphisms $f$ to have a uniform upper bound for the maximal dilatations of their Douady-Earle extensions on a uniform neighborhood of the origin. There are two goals for this paper: one is to introduce some explicit conditions on $f$ such that $f$ satisfies Markovic's criterion; the other goal is to show some explicit conditions on $f$ that do not lead to any upper bound for the maximal 
dilatation of $\Phi(f)$ on any neighborhood of the origin. We have given the statements of these results in the introduction, which are proved in the following sections. Given an arc $\Gamma$ on the unit circle $\mathbf{S}^{1}$, denote by $|\Gamma|$ the arc length of $\Gamma$. Now we end this section by stating Markovic's criterion.

Lemma 1. (Markovic) Let $0<\epsilon<\pi$ and $0<\delta<\pi$. Then there exists an open neighborhood $U(\epsilon, \delta)$ of the origin and a positive constant $M(\epsilon, \delta)$ such that if a circle homeomorphism $f$ satisfies $|f(\Gamma)| \geq \epsilon$ for any arc $\Gamma$ on $\mathbf{S}^{1}$ with $\pi-\delta \leq|\Gamma| \leq \pi$, then $K\left(\left.\Phi(f)\right|_{U(\epsilon, \delta)}\right) \leq M(\epsilon, \delta)$.

Note that it is developed in [6] that the Douady-Earle extension $\Phi(f)$ of a circle homeomorphism $f$ has a local quasiconformality near a circular $\operatorname{arc} \beta$ on $\mathbf{S}^{1}$ if $f$ has a local quasisymmetry on $\beta$.

\section{Proofs of Theorems 1 and 2}

In this section, we develop four propositions to prove Theorems 1 and 2 .

Proposition 1. The supremum $\sup _{f \in \mathcal{F}_{4}^{0}} K(\Phi(f))(0)=\infty$.

Proof. It suffices to show that there exists a sequence $\left\{f_{n}\right\}_{n=1}^{\infty}$ of homeomorphisms in $\mathcal{F}_{4}^{0}$ such that $\lim _{n \rightarrow \infty} K\left(\Phi\left(f_{n}\right)\right)(0)=\infty$.

In the rest of the proof, we assume that $n$ is a positive odd integer. Then $h_{n}(x)=x^{n}$ defines a homeomorphism of the extended real line. Let $g(z)=-i \frac{z-i}{z+i}$, which maps the upper half plane onto the unit disk $\mathbf{D}$ and the extended real line onto the unit circle onto. More specifically, $g$ maps -1 to 1,0 to $i, 1$ to -1 , and $\infty$ to $-i$. Then $f_{n}=g \circ h_{n} \circ g^{-1}$ is a homeomorphism of $\mathbf{S}^{1}$ and fixes four points \pm 1 and $\pm i$. Hence $f_{n} \in F_{4}^{0}$. Since $f_{n}$ is symmetric with respect to the origin (that is, $f_{n}\left(e^{i(\theta+\pi)}\right)=f_{n}\left(e^{i \theta}\right)$ for any $\left.\theta\right), \Phi\left(f_{n}\right)(0)=0$.

The maximal dilatation $K\left(\Phi\left(f_{n}\right)(0)\right)$ of $\Phi\left(f_{n}\right)$ at the origin is equal to $\frac{1-|k(0)|}{1+|k(0)|}$, where $k(0)=\frac{\partial \Phi\left(f_{n}\right)}{\partial \bar{z}}(0) / \frac{\partial \Phi\left(f_{n}\right)}{\partial z}(0)$. Using the expressions (9), (10), (11) and (12), we obtain $k(0)=\frac{c_{-1}+d_{-1} \overline{c_{1}}}{c_{1}+d_{-1} \overline{c_{-1}}}$. In order to understand the asymptotic behavior of $K\left(\Phi\left(f_{n}\right)(0)\right)$ as $n \rightarrow \infty$, we first use (9) and (10) to estimate $c_{1}, c_{-1}, d_{-1}$ for $\Phi\left(f_{n}\right)$. Let $\epsilon=1 / \sqrt{n}$ and let $\xi \in \mathbf{S}^{1}$ be a point on $\mathbf{S}^{1}$ such that $\xi=e^{i \epsilon}$. Then

$$
\xi=e^{i / \sqrt{n}}=1+i \frac{1}{\sqrt{n}}+O\left(\frac{1}{n}\right) .
$$

Thus,

$$
\begin{aligned}
\left(g^{-1}(\xi)\right)^{n} & =\left(\frac{-1-i \xi}{\xi+i}\right)^{n}=\left(\frac{-1-i+1 / \sqrt{n}+O(1 / n)}{1+i+i \sqrt{n}+O(1 / n)}\right)^{n} \\
& =(-1+1 / \sqrt{n}+O(1 / n))^{n}=(-1)^{n}(1-1 / \sqrt{n}+O(1 / n))^{n} \\
& =-\left(\frac{1}{1+1 / \sqrt{n}+O(1 / n)}\right)^{n}=-\left(\frac{1}{(1+1 / \sqrt{n}+O(1 / n))^{\sqrt{n}}}\right)^{\sqrt{n}} \\
& =O\left(e^{-\sqrt{n}}\right) .
\end{aligned}
$$

Hence,

and

$$
f_{n}(\xi)=g\left(O\left(e^{-\sqrt{n}}\right)\right)=-i \frac{O\left(e^{-\sqrt{n}}\right)-i}{O\left(e^{-\sqrt{n}}\right)+i}=i+O\left(e^{-\sqrt{n}}\right)
$$

$$
f_{n}^{2}(\xi)=\left(g\left(O\left(e^{-\sqrt{n}}\right)\right)\right)^{2}=\left(i+O\left(e^{-\sqrt{n}}\right)\right)^{2}=-1+O\left(e^{-\sqrt{n}}\right) .
$$


Note that each map $f_{n}$ is symmetric with respect to the origin. Furthermore, it is symmetric with respect to the $x$-axis and hence to the $y$-axis. Thus, $\bar{z} f_{n}, z f_{n}$ and $f_{n}^{2}$ are symmetric with respect to the $x$-axis and take the same value at $z$ and $-z$ for each $z \in \mathbf{S}^{1}$. Then the quantities $c_{1}, c_{-1}$ and $d_{-1}$ expressed in (9) and (10) can be estimated as follows:

$$
\begin{aligned}
c_{1} & =\frac{1}{2 \pi} \int_{\mathbf{S}^{1}} \bar{z} f_{n}(z)|d z|=\frac{2}{\pi} \operatorname{Re} \int_{0}^{\pi / 2} e^{-i \theta} f_{n}\left(e^{i \theta}\right) d \theta \\
& =\frac{2}{\pi} \operatorname{Re} \int_{0}^{\epsilon} e^{-i \theta} f_{n}\left(e^{i \theta}\right) d \theta+\frac{2}{\pi} \operatorname{Re} \int_{\epsilon}^{\pi / 2} e^{-i \theta} f_{n}\left(e^{i \theta}\right) d \theta \\
& =O(\epsilon)+\frac{2}{\pi} \operatorname{Re} \int_{\epsilon}^{\pi / 2} i e^{-i \theta} d \theta+O\left(e^{-\sqrt{n}}\right) \\
& =O\left(\frac{1}{\sqrt{n}}\right)+\frac{2}{\pi} \operatorname{Re}\left(-e^{-i \frac{\pi}{2}}+e^{-i \epsilon}\right)+O\left(e^{-\sqrt{n}}\right) \\
& =\frac{2}{\pi} \operatorname{Re}\left(i+1-\frac{i}{\sqrt{n}}+O\left(\frac{1}{n}\right)\right)+O\left(\frac{1}{\sqrt{n}}\right)=\frac{2}{\pi}+O\left(\frac{1}{\sqrt{n}}\right), \\
c_{-1} & =\frac{1}{2 \pi} \int_{\mathbf{S}^{1}} z f_{n}(z)|d z|=\frac{2}{\pi} \operatorname{Re} \int_{0}^{\pi / 2} e^{i \theta} f_{n}\left(e^{i \theta}\right) d \theta \\
& =\frac{2}{\pi} \operatorname{Re} \int_{0}^{\epsilon} e^{i \theta} f_{n}\left(e^{i \theta}\right) d \theta+\frac{2}{\pi} \operatorname{Re} \int_{\epsilon}^{\pi / 2} e^{i \theta} f_{n}\left(e^{i \theta}\right) d \theta \\
& =O(\epsilon)+\frac{2}{\pi} \operatorname{Re} \int_{\epsilon}^{\pi / 2} i e^{i \theta} d \theta+O\left(e^{-\sqrt{n}}\right) \\
& =O\left(\frac{1}{\sqrt{n}}\right)+\frac{2}{\pi} \operatorname{Re}\left(e^{i \frac{\pi}{2}}-e^{i \epsilon}\right)+O\left(e^{-\sqrt{n}}\right) \\
& =\frac{2}{\pi} \operatorname{Re}\left(i-1-\frac{i}{\sqrt{n}}+O\left(\frac{1}{n}\right)\right)+O\left(\frac{1}{\sqrt{n}}\right)=\frac{-2}{\pi}+O\left(\frac{1}{\sqrt{n}}\right),
\end{aligned}
$$

and

$$
\begin{aligned}
d_{-1} & =\frac{1}{2 \pi} \int_{\mathbf{S}^{1}} f_{n}^{2}(z)|d z|=\frac{2}{\pi} \operatorname{Re} \int_{0}^{\pi / 2} f_{n}^{2}\left(e^{i \theta}\right) d \theta \\
& =\frac{2}{\pi} \operatorname{Re} \int_{0}^{\epsilon} f_{n}^{2}\left(e^{i \theta}\right) d \theta+\frac{2}{\pi} \operatorname{Re} \int_{\epsilon}^{\pi / 2} f_{n}^{2}\left(e^{i \theta}\right) d \theta \\
& =O(\epsilon)+\frac{2}{\pi} \operatorname{Re} \int_{\epsilon}^{\pi / 2}-1 d \theta+O\left(e^{-\sqrt{n}}\right) \\
& =O\left(\frac{1}{\sqrt{n}}\right)+\frac{2}{\pi}\left(-\frac{\pi}{2}+\epsilon\right)+O\left(e^{-\sqrt{n}}\right)=-1+O\left(\frac{1}{\sqrt{n}}\right) .
\end{aligned}
$$

Then the numerator and denominator of $k(0)$ can be estimated as follows:

$c_{-1}+d_{-1} \overline{c_{1}}=\frac{-2}{\pi}+O\left(\frac{1}{\sqrt{n}}\right)+\left(-1+O\left(\frac{1}{\sqrt{n}}\right)\right)\left(\frac{2}{\pi}+O\left(\frac{1}{\sqrt{n}}\right)\right)=\frac{-4}{\pi}+O\left(\frac{1}{\sqrt{n}}\right)$

and

$c_{1}+d_{-1} \overline{c_{-1}}=\frac{2}{\pi}+O\left(\frac{1}{\sqrt{n}}\right)+\left(-1+O\left(\frac{1}{\sqrt{n}}\right)\right)\left(\frac{-2}{\pi}+O\left(\frac{1}{\sqrt{n}}\right)\right)=\frac{4}{\pi}+O\left(\frac{1}{\sqrt{n}}\right)$. 
Hence,

$$
\left|c_{-1}+d_{-1} \overline{c_{1}}\right| \geq \frac{4}{\pi}-O\left(\frac{1}{\sqrt{n}}\right)
$$

and

$$
\left|c_{1}+d_{-1} \overline{c_{-1}}\right| \leq \frac{4}{\pi}+O\left(\frac{1}{\sqrt{n}}\right) .
$$

Therefore,

$$
k(0) \geq 1-O\left(\frac{1}{\sqrt{n}}\right)
$$

and hence

$$
K\left(\Phi\left(f_{n}\right)\right)(0) \geq \frac{2-O\left(\frac{1}{\sqrt{n}}\right)}{O\left(\frac{1}{\sqrt{n}}\right)} .
$$

This means

$$
\lim _{n \rightarrow \infty} K\left(\Phi\left(f_{n}\right)\right)(0)=\infty .
$$

Remark 1. The collection $\left\{f_{n}\right\}_{n=1}^{\infty}$ of the homeomorphisms $f_{n}$ in the proof of Proposition 1 provides a counter-example to Lemma 2.2 in [10]. By developing a suitable modification of that lemma, it is shown in [4] that the main results of [10] continue to hold.

Proposition 2. The supremum $\sup _{f \in \mathcal{F}_{3}} K(\Phi(f))(0)=\infty$.

Proof. Let $n$ be a positive odd integer and $f_{n}$ be the same as defined in the proof of Proposition 1. Let $A_{n}$ be the conformal homeomorphism of $\mathbf{D}$ fixing 1 and mapping $f_{n}\left(e^{ \pm \frac{2 \pi i}{3}}\right)$ to $e^{ \pm \frac{2 \pi i}{3}}$ respectively. Then $h_{n}=A_{n} \circ f_{n} \in \mathcal{F}_{3}$. From the conformal naturality, $\Phi\left(h_{n}\right)=A_{n} \circ \Phi\left(f_{n}\right)$. Hence, $K\left(\Phi\left(h_{n}\right)\right)(0)=K\left(\Phi\left(f_{n}\right)\right)(0)$. By Proposition 1,

$$
\lim _{n \rightarrow \infty} K\left(\Phi\left(h_{n}\right)\right)(0)=\lim _{n \rightarrow \infty} K\left(\Phi\left(f_{n}\right)\right)(0)=\infty .
$$

Proposition 3. There exists a neighborhood $U$ of the origin in $\mathbf{D}$ such that the supremum

$$
\sup _{f \in \mathcal{F}_{3}^{0}} K\left(\left.\Phi(f)\right|_{U}\right)<\infty
$$

Proof. By the contrapositive of Markovic's Lemma, if our proposition 3 fails, then for any small positive $\epsilon$ and $\delta$, there exist $f \in \mathcal{F}_{3}^{0}$ and an $\operatorname{arc} \Gamma$ on $\mathbf{S}^{1}$ such that $\pi-\delta \leq|\Gamma| \leq \pi$ and $|f(\Gamma)|<\epsilon$, where $|\cdot|$ denote the arc length on $\mathbf{S}^{1}$ with the arc length of $\mathbf{S}^{1}$ equal to $2 \pi$. In particular, for $\epsilon=\delta=1 / n$, there exist $f_{n} \in \mathcal{F}_{3}^{0}$ and an $\operatorname{arc} I_{n} \subset \mathbf{S}^{1}$ such that $\pi-1 / n \leq\left|I_{n}\right| \leq \pi$ and $\left|f_{n}\left(I_{n}\right)\right|<1 / n$. Without loss of generality, we may assume that all $f_{n}$ fix $1, e^{\frac{2 \pi i}{3}}$ and $e^{-\frac{2 \pi i}{3}}$. Since $\left|f_{n}\left(I_{n}\right)\right| \rightarrow 0$ as $n \rightarrow \infty$, both $I_{n}$ and $f_{n}\left(I_{n}\right)$ eventually contain only one of the fixed points. For simplicity and without loss of generality, we may assume that this fixed point is 1 . In the following, we use the condition $\Phi\left(f_{n}\right)(0)=0$ for all $n$ to derive a contradiction. Keep in mind that $\Phi\left(f_{n}\right)(0)=0$ if and only if $\int_{\mathbf{S}^{1}} f_{n}(\zeta)|d \zeta|=0$. On the other hand, 
using the conditions satisfied by $f_{n}$, we can see for large $n$,

$$
\begin{aligned}
\operatorname{Re}\left(\Phi\left(f_{n}\right)(0)\right) & =\operatorname{Re}\left(\int_{\mathbf{S}^{1}} f_{n}(\zeta)|d \zeta|\right) \\
& =\operatorname{Re}\left(\int_{I_{n}} f_{n}(\zeta)|d \zeta|+\int_{J} f_{n}(\zeta)|d \zeta|+\int_{J^{c} \backslash I_{n}} f_{n}(\zeta)|d \zeta|\right) \\
& \geq \cos (\epsilon)\left(\frac{1}{2}-\frac{\delta}{\pi}\right)+\frac{1}{3}(-1)+\left(\frac{1}{6}+\frac{\delta}{\pi}\right)\left(-\frac{1}{2}\right) \\
& =\cos \left(\frac{1}{n}\right)\left(\frac{1}{2}-\frac{1}{n \pi}\right)-\frac{1}{3}-\frac{1}{12}-\frac{1}{2 n \pi},
\end{aligned}
$$

where $J$ is the short arc on $\mathbf{S}^{1}$ between $e^{\frac{2 \pi i}{3}}$ and $e^{-\frac{2 \pi i}{3}}$ and $J^{c}=\mathbf{S}^{1} \backslash J$. Thus

$$
\lim _{n \rightarrow \infty} \operatorname{Re}\left(\Phi\left(f_{n}\right)(0)\right)=1 / 12>0 .
$$

Hence $\Phi\left(f_{n}\right)(0) \neq 0$ for large $n$, which is a contradiction. Therefore, our Proposition 3 holds.

Finally, we prove the case $\mathcal{F}_{n}$ for $n \geq 5$. that

Proposition 4. There exists a universal neighborhood $U$ of the origin in $\mathbf{D}$ such

$$
\sup _{n \geq 5} \sup _{f \in \mathcal{F}_{n}} K\left(\left.\Phi(f)\right|_{U}\right)<\infty .
$$

Proof. Let $n \geq 5, f \in \mathcal{F}_{n}$ and $P_{n}$ the collection of $n$ points evenly spread on $\mathbf{S}^{1}$ and fixed by $f$. Let $\Gamma$ be an arc with arc length $\pi-\frac{\pi}{5}=\frac{4 \pi}{5}<|\Gamma|<\pi$. By checking the number of fixed points of $f$ contained in $\Gamma$, one can obtain $|f(\Gamma)| \geq \frac{2 \pi}{7}$ and in fact the lower bound $\frac{2 \pi}{7}$ is attained when $f \in \mathcal{F}_{7}$. This claim can be proved by considering three cases as follows.

Case 1. When $5 \leq n \leq 7,|\Gamma|>\frac{4 \pi}{5} \geq 2\left(\frac{2 \pi}{n}\right)$. This implies that $\Gamma$ contains at least two adjacent points of $P_{n}$ and then $|f(\Gamma)| \geq \frac{2 \pi}{n} \geq \frac{2 \pi}{7}$.

Case 2. When $8 \leq n \leq 9,|\Gamma|>\frac{4 \pi}{5} \geq 3\left(\frac{2 \pi}{n}\right)$. This implies that $\Gamma$ contains at least three consecutive points of $P_{n}$ and then $|f(\Gamma)| \geq 2 \frac{2 \pi}{n}>\frac{2 \pi}{7}$.

Case 3. When $n \geq 10$, let $n=5 k+j$, where $k \geq 2$ and $0 \leq j \leq 4$. Then

$$
\frac{4 \pi}{5} / \frac{2 \pi}{n}=\frac{2 n}{5}=\frac{2(5 k+j)}{5} \geq 2 k \text {. }
$$

It follows that $\Gamma$ contains at least $2 k$ consecutive points of $P_{n}$. Then

$$
|f(\Gamma)| \geq(2 k-1) \frac{2 \pi}{n}=2 \pi \frac{2 k-1}{5 k+j}=2 \pi \frac{2-1 / 5}{5+j / k}>\frac{2 \pi}{7}
$$

because $k \geq 2$ and $0 \leq j \leq 4$.

Therefore, all maps $f \in \cup_{n=5}^{\infty} \mathcal{F}_{n}$ satisfy the condition required in Markovic's Lemma for $\epsilon=\frac{2 \pi}{7}$ and $\delta=\frac{\pi}{5}$. Hence there exist a neighborhood $U(\delta)$ and a constant $M(\epsilon, \delta)$ such that $K\left(\left.\Phi(f)\right|_{U(\delta)}\right)<M(\epsilon, \delta)$ for any $f \in \mathcal{F}_{n}$. Proposition 4 follows.

Theorem 2 follows from Proposition 3, Proposition 1 and the fact that $\mathcal{F}_{4}^{0} \subset \mathcal{F}_{1}^{0}$ and $\mathcal{F}_{4}^{0} \subset \mathcal{F}_{2}^{0}$.

Theorem 1 follows from Proposition 4, Proposition 2, Proposition 1 and the fact that $\mathcal{F}_{4}^{0} \subset \mathcal{F}_{k}$ for each $k=1,2,4$. 


\section{Proof of Theorem 4}

Assume $n \geq 4$. Let $S_{n}$ be a set of $n$ points evenly distributed on $\mathbf{S}^{1}$ and a quadruple $Q \subset S_{n}$. Define

$$
L(n)=\max _{Q \subset S_{n}} \operatorname{cr}(Q) .
$$

Then $L(n) \geq 1$ and $L(n) \rightarrow \infty$ as $n \rightarrow \infty$. In fact, one can prove that $L(n)$ is an increasing function of $n$. Let $p_{j}=e^{\frac{2 \pi j}{n} i}, j=0,1, \cdots, n-1$, and let $k=\left\lfloor\frac{n}{2}\right\rfloor$ (the largest integer less than or equal to $\frac{n}{2}$ ). Then

$$
\frac{1}{L(n)}=\operatorname{cr}\left(\left\{p_{0}, p_{1}, p_{k}, p_{k+1}\right\}\right)=\frac{\left|p_{0}-p_{1}\right|^{2}}{\left|p_{0}-p_{k+1}\right|^{2}}=\left[\frac{\sin \frac{\pi}{n}}{\sin \frac{(k+1) \pi}{n}}\right]^{2} \text {. }
$$

If $n$ is even, then

$$
\frac{1}{L(n)}=\left[\frac{\sin \frac{\pi}{n}}{\cos \frac{\pi}{n}}\right]^{2}=\left[\tan \frac{\pi}{n}\right]^{2}
$$

If $n$ is odd, then

$$
\frac{1}{L(n)}=\left[\frac{\sin \frac{\pi}{n}}{\cos \frac{\pi}{2 n}}\right]^{2}=\left[2 \sin \frac{\pi}{2 n}\right]^{2} .
$$

Now it is clear to see that $\frac{1}{L(n)}$ is decreasing when $n$ is even or odd. To claim that $\frac{1}{L(n)}$ is decreasing for all $n$, we consider $\sqrt{\frac{1}{L(n)}}$. It suffices to show for any integer $m \geq 2$,

$$
\tan \frac{\pi}{2 m+2}<2 \sin \frac{\pi}{2(2 m+1)}<\tan \frac{\pi}{2 m} .
$$

This double inequality can be verified by using the Taylor series of sin and tan at 0 .

In the following, we first prove a lemma, which is a subcase of Theorem 4 and in the meantime provides the motivation and essence to prove Theorem 4. Before proving this lemma and Theorem 4, we clarify a terminology used in the proofs of these results. Let $n \geq 5$ and $P$ be a collections of $n$ points on the unit circle. Given $2 \leq k \leq n-1$, by a circular arc spanned by $k$ consecutive points of $P$ we mean the shortest arc containing exactly these points.

Lemma 2. For any $M>0$, there exists a neighborhood $U$ of the origin in $\mathbf{D}$ depending on $M$ such that

$$
\sup _{f \in \mathcal{A}_{5}^{L(5), M}} K\left(\left.\Phi(f)\right|_{U}\right)<\infty .
$$

Note that $\mathcal{A}_{5}^{L(5), M}=\mathcal{B}_{5}^{M}$. Hence this lemma proves the conclusion of Theorem 3 for the case $n=5$.

Proof. Let $L=L(5)$ and $f \in \mathcal{A}_{5}^{L, M}$. Using the conformal natural property of Douady-Earle extension $\Phi(f)$ of $f$ and the property that any conformal homeomorphism of $\mathbf{D}$ preserves the cross ratio of any quadruple, we may assume that $\Phi(f)(0)=0$ by post-composing $f$ by a conformal homeomorphism of $\mathbf{D}$.

Let $a, b, c, d$ and $e$ be five points evenly distributed on $\mathbf{S}^{1}$ in the counterclockwise order and let $P$ denote the collection of these five points. Denote by $a^{\prime}, b^{\prime}, c^{\prime}, d^{\prime}$ and $e^{\prime}$ the images of the five points under $f$ respectively. Note that for any quadruple $Q \subset P, \frac{1}{L} \leq \operatorname{cr}(Q) \leq L$. Then by definition, $\operatorname{cr}(f(Q)) \geq \frac{1}{L} e^{-M}$.

The proof of this lemma is divided into 4 steps. In the first step, we show that the Lebesque measure of the image of any closed arc containing 4 consecutive points 
in $P$ is bounded from below away from zero. In the second and third steps, we show similar bounds for closed arcs containing 3 and 2 consecutive points in $P$ respectively. Finally, in the last step we apply Markovic's Lemma to deduce the conclusion.

Step 1. We show that there exists a constant $\rho>0$ such that for any arc $I$ on $\mathbf{S}^{1}$ spanned by four (adjacent) points of $P$, the Lebesque measure $m(f(I))>2 \rho>0$. Without loss of generality, we may assume that $I$ is the arc containing $b$ and $c$ with endpoints at $a$ and $d$. Because of the conformal naturality and invariance of cross ratio under rotation, we can postcompose $f$ by a rotation such that the real parts of $\operatorname{Re}(f(a))$ and $\operatorname{Re}(f(d))$ are the same, which is denoted by $x$. We continue to use $f$ to denote the map. Note that the normalization condition $\Phi(f)(0)=0$ is also preserved under postcomposition by a rotation. Then $\int_{\mathbf{S}^{1}} \operatorname{Re}(z) d \mu_{f}(z)=0$, where $\mu_{f}$ is the push-forward of the Lebesgue measure $m$ on $\mathbf{S}^{1}$ by $f$. On the other hand,

$$
\begin{aligned}
\int_{\mathbf{S}^{1}} \operatorname{Re}(z) d \mu_{f}(z) & \geq(-1) \mu_{f}\left(\widehat{d^{\prime} e^{\prime} a^{\prime}}\right)+x \mu_{f}\left(\widehat{a^{\prime} b^{\prime} c^{\prime} d^{\prime}}\right) \\
& =(-1) m(\widehat{d e a})+(x) m(\widehat{a b c d})=-\frac{2}{5}+x \frac{3}{5},
\end{aligned}
$$

where $\widehat{u \cdots v}$ denotes the arc on $\mathbf{S}^{1}$ from $u$ to $v$ in the counterclockwise direction. Then $-\frac{2}{5}+x \frac{3}{5} \leq 0$ and hence $x \leq 2 / 3$. Therefore, $m(f(I)) \geq 2 \arccos (2 / 3)$. Letting $\rho=\arccos (2 / 3)$, we obtain the claim at the beginning.

Step 2. We show that there exists a constant $\rho_{1}>0$ such that for any arc $I$ spanned by three adjacent points of $P, m(f(I)) \geq \rho_{1}$. Without loss of generality, we take $I=\widehat{b c d}$. Let $\rho$ be the constant obtained in Step 1, We show if $m\left(\widehat{b^{\prime} c^{\prime} d^{\prime}}\right)<\rho$, then $m\left(\widehat{b^{\prime} c^{\prime} d^{\prime}}\right)$ is greater than or equal to another positive constant. Assume that $m\left(\widehat{b^{\prime} c^{\prime} d^{\prime}}\right)<\rho$. Using the conclusion of Step 1, we can see that both $m\left(\widehat{d^{\prime} e^{\prime}}\right)$ and $m\left(\widehat{a^{\prime} b^{\prime}}\right)$ are greater than $2 \rho-m\left(\widehat{b^{\prime} c^{\prime} d^{\prime}}\right)>\rho$. Therefore, $\left|d^{\prime} e^{\prime}\right|,\left|a^{\prime} b^{\prime}\right|>2 \sin (\rho / 2)$. Let $Q$ be the quadruple of $e, a, b$ and $d$. Using the lower bound of $\operatorname{cr}(f(Q))$, we obtain

$$
\frac{1}{L} e^{-M} \leq \frac{\left|e^{\prime} a^{\prime}\right|\left|b^{\prime} d^{\prime}\right|}{\left|a^{\prime} b^{\prime}\right|\left|e^{\prime} d^{\prime}\right|} \leq \frac{2\left|b^{\prime} d^{\prime}\right|}{4 \sin ^{2}(\rho / 2)}
$$

Thus,

$$
\left|b^{\prime} d^{\prime}\right| \geq 2 \frac{1}{L} e^{-M} \sin ^{2}(\rho / 2) .
$$

Let $\rho^{\prime}=2 \arcsin \left(\frac{1}{L} e^{-M} \sin ^{2}(\rho / 2)\right)$. Then we have shown $m\left(\widehat{b^{\prime} c^{\prime} d^{\prime}}\right) \geq \rho^{\prime}$ if $m\left(\widehat{b^{\prime} c^{\prime} d^{\prime}}\right)<$ $\rho$. Now we let $\rho_{1}=\min \left\{\rho, \rho^{\prime}\right\}$. Then $m\left(\widehat{b^{\prime} c^{\prime} d^{\prime}}\right) \geq \rho_{1}$ always.

Step 3. We show that there exists a constant $\rho_{2}>0$ such that for any arc $I$ spanned by two adjacent points of $P$, the Lebesque measure $m(f(I)) \geq \rho_{2}$. Without loss of generality, we take $I=\widehat{b c}$. Let $\rho_{1}$ be the constant obtained in Step 2. We show if $m\left(\widehat{b^{\prime} c^{\prime}}\right)<\rho_{1} / 2$, then $m\left(\widehat{b^{\prime} c^{\prime}}\right)$ is greater than or equal to another positive constant. Assume that $m\left(\widehat{b^{\prime} c^{\prime} d^{\prime}}\right)<\rho_{1} / 2$. Using the conclusion of Step 2, we can see that both of $m\left(\widehat{c^{\prime} d^{\prime}}\right)$ and $m\left(\widehat{a^{\prime} b^{\prime}}\right)$ are greater than $\rho_{1}-m\left(\widehat{b^{\prime} c^{\prime}}\right)>\rho_{1} / 2$. Then $\left|d^{\prime} e^{\prime}\right|,\left|a^{\prime} b^{\prime}\right|>2 \sin \left(\rho_{1} / 4\right)$. Let $Q$ be the quadruple of $b, c, d$ and $a$. Using the lower bound of $\operatorname{cr}(f(Q))$, we obtain

$$
\frac{1}{L} e^{-M} \leq \frac{\left|b^{\prime} c^{\prime}\right|\left|d^{\prime} a^{\prime}\right|}{\left|c^{\prime} d^{\prime}\right|\left|a^{\prime} b^{\prime}\right|} \leq \frac{2\left|b^{\prime} c^{\prime}\right|}{4 \sin ^{2}\left(\rho_{1} / 4\right)}
$$


Thus,

$$
\left|b^{\prime} c^{\prime}\right| \geq 2 \frac{1}{L} e^{-M} \sin ^{2}\left(\rho_{1} / 4\right)
$$

Let $\rho^{\prime \prime}=2 \arcsin \left(\frac{1}{L} e^{-M} \sin ^{2}\left(\rho_{1} / 4\right)\right)$. Then we have shown $m\left(\widehat{b^{\prime} c^{\prime}}\right) \geq \rho^{\prime \prime}$ if $m\left(\widehat{b^{\prime} c^{\prime}}\right)<$ $\rho_{1} / 2$. Overall, $m\left(\widehat{b^{\prime} c^{\prime}}\right) \geq \rho_{2}$ for $\rho_{2}=\min \left\{\rho_{1} / 2, \rho^{\prime \prime}\right\}$. Hence, $m(f(I)) \geq \rho_{2}$.

Step 4 . Let $\Gamma$ be a closed arc on $\mathbf{S}^{1}$ with arc length $\pi-\pi / 5 \leq|\Gamma| \leq \pi$. Then $\Gamma$ contains two adjacent points in $P$ and hence $m(f(\Gamma)) \geq \rho_{2}$ by using the conclusion of Step 3. By setting $\delta=\pi / 5$ and $\epsilon=\rho_{2}$ obtained in Step 3 and applying Markovic's Lemma, we conclude that there exists a constant $C>0$ and a neighborhood $U$ of the origin such that $K\left(\left.\Phi(f)\right|_{U}\right) \leq C$.

An immediate corollary follows as:

Corollary 2. If $n$ is a multiple of 5 , then $\mathcal{A}_{n}^{L(n), M} \subset \mathcal{A}_{5}^{L(5), M}$ and hence

$$
\sup _{f \in \mathcal{A}_{n}^{L(n), M}} K\left(\left.\Phi(f)\right|_{U}\right) \leq \sup _{f \in \mathcal{A}_{5}^{L(5), M}} K\left(\left.\Phi(f)\right|_{U}\right)<\infty,
$$

where $U$ is the same neighborhood of the origin given in the previous lemma.

Proof of Theorem 4. Let $M$ be a positive constant. Using Markovic's criterion (Lemma 1), we only need to prove there exist three positive constants $L, \delta$ and $\epsilon$ such that for any $f \in \bigcup_{n=5}^{\infty} \mathcal{A}_{n}^{L, M}$,

$$
|f(\Gamma)| \geq \epsilon
$$

for any arc $\Gamma$ on $\mathbf{S}^{1}$ with $\pi-\delta \leq|\Gamma| \leq \pi$. We divide the work into two steps.

Step 1. Assume $f \in \mathcal{A}_{n}^{L(n), \bar{M}}$ for some $5 \leq n \leq 20$, where $L(n)$ is the same as defined at the beginning of this section. We prove that there exists $\epsilon_{n}$ such that for any $\operatorname{arc} \Gamma$ with $\frac{4 \pi}{n} \leq|\Gamma| \leq \pi, m(f(\Gamma)) \geq \epsilon_{n}$.

Note that we have obtained the result for the case $n=5$ in Lemma 2, which we include here to have uniform notation for all cases of this step. In fact, the main strategies and techniques to achieve the result of this step for any other case $5<n \leq 20$ are very similar to the ones used to handle the case $n=5$.

At first, we may assume $\Phi(f)(0)=0$ by postcomposing $f$ by a Mob̈ius transformation preserving $\mathbf{D}$. Let $P=\left\{a_{1}, a_{2}, a_{3}, \cdots, a_{n}\right\}$ be a collection of $n$ points evenly spread on the unit circle and arranged in the counterclockwise direction. Assume $l=\left\lfloor\frac{n}{2}+2\right\rfloor$ (the largest integer less than or equal to $\left.\frac{n}{2}+2\right)$.

(1) Let $I$ be an arc spanned by $l$ consecutive points in $P$. Then $m(I)$ is a constant strictly greater than $\frac{1}{2}$. Using the normalization condition $\Phi(f)(0)=0$ and the strategy used in Step 1 in the proof of Lemma 2, we can show that $m(f(I))$ is greater than or equal to a positive constant $\rho$ only depending on $m(I)$.

(2) Let $I$ be an arc spanned by $l-1$ consecutive points of $P$. Without loss of generality, we let $I=a_{2}, \widehat{a_{3}, \cdots}, a_{l}$. By using the conclusion of (1), we know $m\left(f\left(a_{1}, \widehat{a_{2}, \cdots}, a_{l}\right)\right)$ and $m\left(f\left(a_{2}, \widehat{a_{3}, \cdots}, a_{l+1}\right)\right)$ are greater than or equal to $\rho$. Then using the lower bound of the cross ratio $\operatorname{cr}\left(f\left(\left\{a_{2}, a_{l}, a_{l+1}, a_{1}\right\}\right)\right)$ and the same strategy used in Step 2 in the proof of Lemma 2, we can obtain another positive constant $\rho_{1}$ such that $m(f(I)) \geq \rho_{1}$.

(3) Let $I$ be an arc spanned by $l-2$ consecutive points of $P$. By using other two points of $P$ adjacent to $I$ and the same strategy of (2), we can obtain a positive constant $\rho_{2}$ such that $m(f(I)) \geq \rho_{2}$. Using this idea inductively to reduce the number of consecutive points in $P$ spanning $I$, we can obtain positive constants $\rho_{3}, \rho_{4}, \cdots, \rho_{l-2}$ such that for any arc $I$ spanned by $l-j$ consecutive points of $P$, 
$m(f(I)) \geq \rho_{j}$, where $j=3,4, \cdots, l-2$. Thus, we obtain for any arc spanned by two adjacent points of $P, m(f(I)) \geq \rho_{l-2}$.

(4) Now let $\epsilon_{n}=\rho_{l-2}$ obtained in (3). Then, given any arc $\Gamma$ on $\mathbf{S}^{1}$ with $\frac{4 \pi}{n} \leq$ $|\Gamma| \leq \pi, \Gamma$ contains at least two adjacent points of $P$. Using the conclusion of (3), we obtain $m(f(I)) \geq \epsilon_{n}$. We denote by $\delta_{n}=\pi-\frac{4 \pi}{n}$. Then $\pi-\delta_{n} \leq|\Gamma| \leq \pi$. Note also that $\delta_{n}>\frac{\pi}{10}$ for each $5 \leq n \leq 20$.

Step 2. We prove there exist positive constants $L>1$ and $\epsilon^{\prime}>0$ such that for any $n \geq 21, f \in \mathcal{A}_{n}^{L, M}$, and any circular arc $\Gamma$ with $\pi-\frac{\pi}{10}<|\Gamma|<\pi, m(f(\Gamma)) \geq \epsilon^{\prime}$.

Now we assume $n \geq 21$ and $P_{n}$ is a collection of $n$ points evenly spread on $\mathbf{S}^{1}$. For each $0 \leq j \leq 4$, there is a point $p_{j} \in P_{n}$ such that the arc distance between $p_{j}$ and $e^{\frac{2 \pi j}{5}}$ is less than or equal to $\frac{\pi}{n}$, which is less than $\frac{\pi}{20}$ (since $n \geq 21$ ). Then $P_{n}^{\prime}=\left\{p_{j}: j=0,1, \cdots, 4\right\}$ consists of five distinct points of $P_{n}$. It is easy to see that $\max _{Q \subset P_{n}^{\prime}} \operatorname{cr}(Q)$ converges to $L(5)$ as $n \rightarrow \infty$. In fact, the arc length between any two adjacent points in $P_{n}^{\prime}$ is greater than $\frac{2 \pi}{5}-\frac{\pi}{10}>\frac{2 \pi}{10}$. It follows that

$$
\max _{Q \subset P_{n}^{\prime}} \operatorname{cr}(Q)<L(10)<L(20) \text {. }
$$

Now we let

$$
L=\sup _{n \geq 21}\left(\max _{Q \subset P_{n}^{\prime}} \operatorname{cr}(Q)\right) .
$$

Clearly, $L<L(20)$. Then for each $f \in \mathcal{A}_{n}^{L, M}$ with $n \geq 21$, we first normalize $\Phi(f)(0)=0$ by post-composing $f$ by a Möbius transformation. Then we apply Steps 1-3 in the proof of Lemma 2 to the map $f$ restricted on $P_{n}^{\prime}$ and obtain a positive constant $\epsilon^{\prime}$ such that for any arc $I$ spanned two adjacent points of $P_{n}^{\prime}, m(f(I)) \geq \epsilon^{\prime}$.

Now we show given any circular arc $\Gamma$ with $\pi-\frac{\pi}{10}<|\Gamma|<\pi, m(f(I)) \geq \epsilon^{\prime}$. It suffices to know that $\Gamma$ contains at least two points of $P_{n}^{\prime}$, which is enough to show $|\Gamma|$ is greater than or equal to the maximum of the arc lengths of the arcs $\widehat{a b c}$ spanned by three consecutive points of $P_{n}^{\prime}$. This is true from how $P_{n}^{\prime}$ is constructed; that is,

$$
|\widehat{a b c}| \leq \frac{4 \pi}{5}+\frac{\pi}{20}+\frac{\pi}{20}=\frac{9 \pi}{10}<|\Gamma|
$$

Now we use the conclusions of Step 1 and Step 2 to complete the proof. Let $\epsilon=\min \left\{\epsilon_{5}, \ldots, \epsilon_{20}, \epsilon^{\prime}\right\}$ and let $L$ be as the same as the one defined in Step 2 (note that one may take $L=L(20))$. Then for any $f \in \bigcup_{n \geq 5} \mathcal{A}_{n}^{L, M}$ and any arc $\Gamma$ with $\pi-\frac{\pi}{10}<|\Gamma|<\pi$

$$
m(f(\Gamma))>\epsilon .
$$

Therefore by Markovic's Lemma, there exist a constant $C>0$ and a neighborhood $U$ of the origin in $\mathbf{D}$ such that for all $f \in \cup_{n \geq 5} \mathcal{A}_{n}^{M}$,

$$
K\left(\left.\Phi(f)\right|_{U}\right)<C .
$$

Acknowledgement. Both authors wish to thank the referee for his/her comments and corrections of typos.

\section{References}

[1] Abikoff, W.: Conformal barycenters and the Douady-Earle extension. A discrete dynamical approach. - J. Anal. Math. 86, 2002, 221-234.

[2] Abikoff, W., C. Earle, and S. Mitra: Barycentric extensions of monotone maps of the circle. - Contemp. Math. 355, 2004, 1-20.

[3] Doundy, A., and C. EARLE: Conformally natural extension of homeomorphisms of the circle. - Acta Math. 157, 1986, 23-48. 
[4] FAn, J., and J. Hu: Characterization of the asymptotic Teichmüller space of the open unit disk through shears. - Pure Appl. Math. Q. 10:3, 2014, 513-46.

[5] Hu, J., and O. Muzician: Cross-ratio distortion and Douady-Earle extension: I. A new upper bound on quasiconformality. - J. London Math. Soc. 86:2, 2012, 387-406.

[6] Hu, J., and O. Muzician: Cross-ratio distortion and Douady-Earle extension: II. Quasiconformality and asymptotic conformality are local. - J. Anal. Math. 117:1, 2012, 249-271.

[7] Hu, J., and O. Muzician: Conformally natural extensions of continuous circle maps: I. The case when the pushforward measure has no atom. - Contemp. Math. 575, 2012, 171-198.

[8] Hu, J., and O. Muzician: Conformally natural extensions of continuous circle maps: II. The general case. - J. Anal. Math. 132, 2017, 81-207.

[9] Markovic, V.: Quasisymmetric groups. - J. Amer. Math. Soc. 19, 2006, 673-715.

[10] ŠArIĆ, D.: Circle homeomorphisms and shears. - Geom. Topol. 14, 2010, 2405-2430.

Received 6 January 2018 • Accepted 10 December 2018 